\title{
Antiwindup Analysis and Design Approaches for MIMO Systems
}

Vincent R. Marcopoli and Stephen M. Phillips

Case Western Reserve University

Cleveland, Ohio

April 1994

Prepared for

Lewis Research Center

Under Grant NAG3-1232

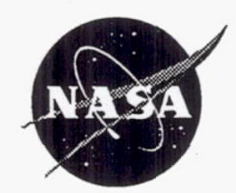

National Aeronautics and Space Administration 


\title{
ANTIWINDUP ANALYSIS AND DESIGN APPROACHES FOR MIMO SYSTEMS
}

\author{
Vincent R. Marcopoli and Stephen M. Phillips * \\ Case Western Reserve University \\ Cleveland, $\mathrm{OH} 44106$
}

\begin{abstract}
Performance degradation of multiple-input multipleoutput (MIMO) control systems having limited actuators is often handled by augmenting the controller with an antiwindup mechanism, which attempts to maintain system performance when limits are encountered. The goals of this paper are: 1) To develop a method to analyze antiwindup systems to determine precisely what stability and performance degradation is incurred under limited conditions. It is shown that by reformulating limited actuator commands as resulting from multiplicative perturbations to the corresponding controller requests, $\mu$-analysis tools can be utilized to obtain quantitative measures of stability and performance degradation. 2) To propose a linear, time invariant (LTI) criterion on which to base the antiwindup design. These analysis and design methods are illustrated through the evaluation of two competing antiwindup schemes augmenting the controller of a Short Take-Off and Vertical Landing (STOVL) aircraft in transition flight.
\end{abstract}

\section{Introduction}

Control design for actuator-limited plants is commonly dealt with using a two-step design approach. First, a linear control design is completed for nominal operating conditions, ignoring limits. The controller is then augmented with a strategy to prevent actuator windup should one or more control requests violate the limits. Since the antiwindup scheme is not generated from the control design, its effect on overall system performance is unknown. Much of the literature on this subject evaluates antiwindup performance heuristically by displaying well-behaved simulation time histories of the system response. This work presents a method of quantitatively assessing closed

-This work was supported in part by the Advanced Control Technology Branch of the NASA Lewis Research Center through grant NAG3-1232. loop stability and performance degradation properties of antiwindup systems using $\mu$-analysis tools. Finally, preliminary work is shown which suggests an LTI criterion for antiwindup system design.

\section{System Description}

\subsection{Nominal Control Design}

The problem of limited actuators is addressed here via the integrated flight control for interconnected propulsion and airframe subsystems. A controller is first obtained for the nominal plant: a simplified linear model of a supersonic Short Take-Off and Vertical Landing (STOVL) aircraft in transition flight, which is unstable. The nominal linear integrated flight/propulsion model consists of the longitudinal flight dynamics (5th order), propulsion dynamics (2nd order), and actuator dynamics (8 first order actuators). There are four "regulated" output variables, $y_{-} r$, and six "measured" output variables, $y_{-} m$, in addition to the four tracking errors, $e$. The nominal design plant is shown in Figure 1, depicting the complete assignments of exogenous $(w)$ and actuator $\left(u_{-} c\right)$ inputs, regulated $(z)$ and measured $(y)$ outputs, and frequency dependent weighting functions which establish performance and stability robustness specifications [1]. The nominal controller is obtained via $H_{\infty}$ optimization of the design plant closed loop transfer function from $w$ to $z, H_{z w}$ [2].

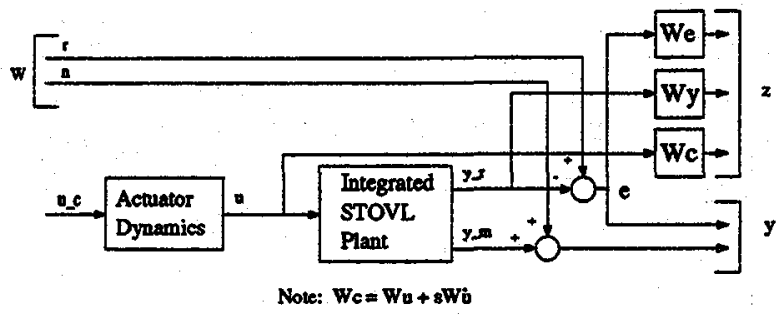

Figure 1: $H_{\infty}$ design plant 


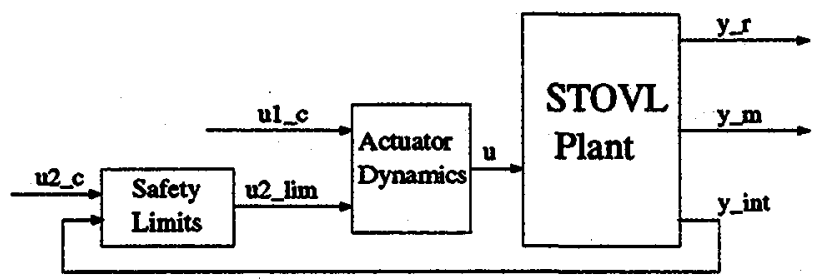

Figure 2: Limited STOVL Plant

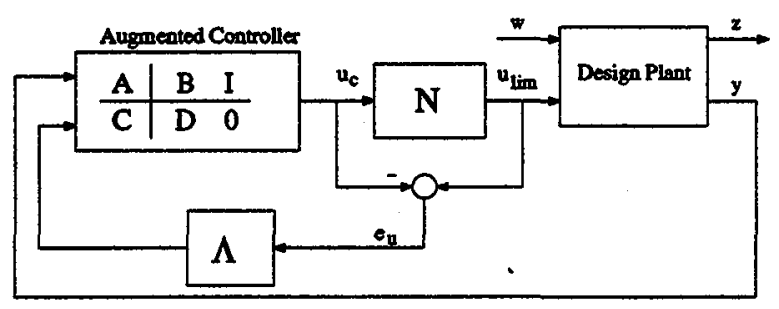

Figure 3: Closed Loop Antiwindup System

\subsection{Actuator Limits}

Actuator limitations arise from propulsion system safety requirements. There are two safety protection mechanisms in the integrated system:

1. Constraints on fuel flow $(W F)$. Both upper and lower fuel flow limits ensure proper pressure, temperature, and fan speed levels in the turbomachinery.

2. Constraints on nozzle area actuators, (the aft and ventral nozzle areas $A 8$ and $A 78$ ), and the ejector butterfly valve angle $(E T A)$. ETA controls the nozzle area of the ejectors, producing vertical thrust. Lower limits are imposed on the total area to prevent surge conditions, which result when the airflow through the compressor and fan blades is excessively restricted.

The above limits are determined via nonlinear feedback of appropriate engine quantities that indicate proximity of the engine to unsafe operating conditions. A schematic representation of the limited system is shown in Figure 2.

\subsection{Antiwindup System}

The closed-loop limited antiwindup system is shown in Figure 3. The element $N$ is the limiting nonlinearity, which modifies the controller commands if upper or lower limits are reached. In many systems this block is a saturation-type nonlinearity. This is the case for the current STOVL system example, however, the upper and lower limits vary according to flight conditions.

The nominal controller has the state space realization $(A, B, C, D)$. The "augmented" controller shown in Figure 3 has the realization $\left(A,\left[\begin{array}{ll}B & I\end{array}\right], C,\left[\begin{array}{ll}D & 0\end{array}\right]\right)$, and allows a memoryless matrix gain, $\Lambda$, to modify the nominal controller states based on the difference between the limited and nonlimited actuator values, $e_{u}$. This block was first introduced in [3], and both generalizes and parameterizes multivariable antiwindup schemes. In this context, antiwindup design reduces to determining a suitable choice for $\Lambda$.

Two choices for $\Lambda$ will be considered here, corresponding to two antiwindup approaches. Referring to Figure 3, one can view the antiwindup scheme as a feedback loop around the augmented controller. It can be shown that the feedback parameter $\Lambda$ modifies the augmented controller state equations as follows:

$$
\begin{aligned}
\dot{x} & =(A-\Lambda C) x+(B-\Lambda D) y+\Lambda u_{l i m} \\
u_{c} & =C x+D y
\end{aligned}
$$

With this view one of the goals of the antiwindup design is to make these modified dynamics relatively fast and well damped in order to regulate to zero any differences between the limited actuator signals, $u_{\text {lim }}$, and the actuator signals commanded by the controller, $u_{c}$. Note that without the antiwindup protection, integrators present in the nominal controller will not allow successful regulation of the difference between these signals.

For this paper one choice of $\Lambda$ is to place the eigenvalues of $A-\Lambda C$ further to the left by choosing $\Lambda$ to attempt to make $A-\Lambda C=A-k I$ [4]. Experiments show that $k=10$ yields desirable antiwindup performance. Due to the dimensions of the system, this cannot be solved exactly (not enough degrees of freedom in $\Lambda$ ). Thus a least squares approach is used to solve $\Lambda_{2} C=k I$. Strictly for the purposes of comparison another choice of $\Lambda$ was the least squares solution of $C \Lambda_{1}=k I$. As expected, the performance of $\Lambda_{2}$ is superior and is shown below.

\subsection{Step Response}

Figures 4-7 illustrate time responses of three configurations of the closed loop system shown in Figure 3 to a step of 3 in the $\gamma$ reference command, with the remaining three reference commands held at zero. This reference command was chosen because it is essentially a propulsive lift command to the aircraft, and drives the fuel flow and nozzle areas to their respective safety limits. Specifically, Figure 4 compares the decoupled $\gamma$-tracking capability of the nominal linear system with no limits present (solid line) with that of the two limited antiwindup systems discussed in the previous section: $\Lambda=\Lambda_{1}$ (dashed line) and $\Lambda=\Lambda_{2}$ (dotted line). In the sequel, these two antiwindup systems will be denoted AW1 and AW2, respectively. Note that the limited systems are characterized by oscillatory behavior and loss of decoupling, with respect to the nominal linear system. Note also that 


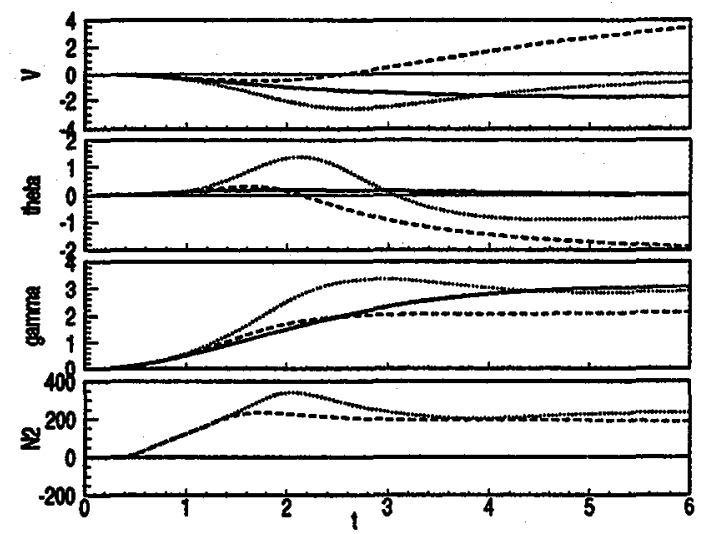

Figure 4: Decoupled command tracking step response comparison

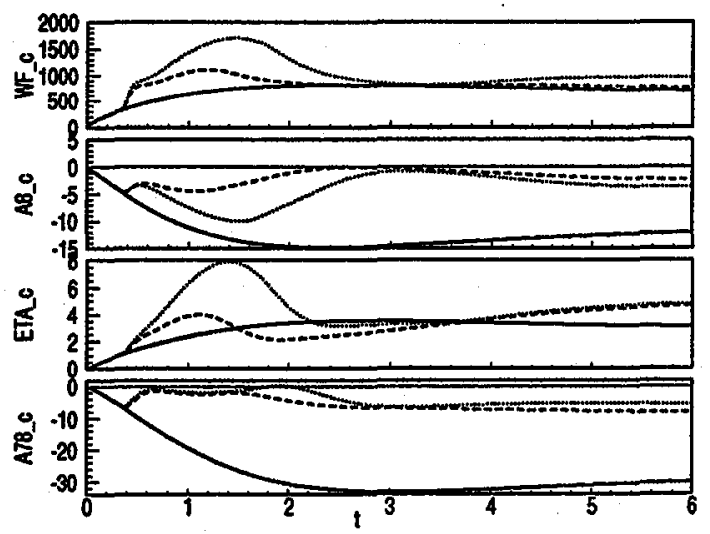

Figure 5: Controller output step response comparison

AW1 results in a significantly greater steady-state $\gamma$ tracking error compared with AW2.

Figure 5 compares the actuator commands of the three systems. The antiwindup systems have the characteristic of boosting the fuel flow command in response to the area limit becoming active. This action occurs at approximately 0.4 seconds. This is seen more clearly in Figures 6-7, which compare the actual commands given to the plant (solid lines), with the controller output (dashed lines) for AW1 (Figure 6) and AW2 (Figure 7). Note that the fuel flow boost actually causes the fuel flow limit (dotted line) to be exceeded.

From the above simulations, it is apparent that AW2 is in some sense "better" than AW1, because of the steady-state $\gamma$ tracking error and no significant differences in any other responses. Most literature on antiwindup schemes stops with this heuristic evaluation. However, for a general antiwindup design method, a quantitative distinction between competing schemes is required, in order for the "best" antiwindup scheme to be obtained.

It is the goal of this paper to formulate an LTI cost criterion on which to base the selection of $\Lambda$. This is
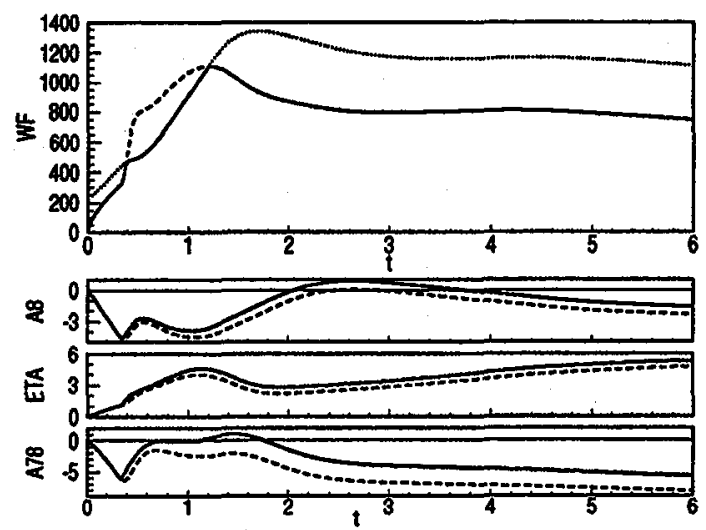

Figure 6: Comparison of controller commands with limited actuator commands for AW1

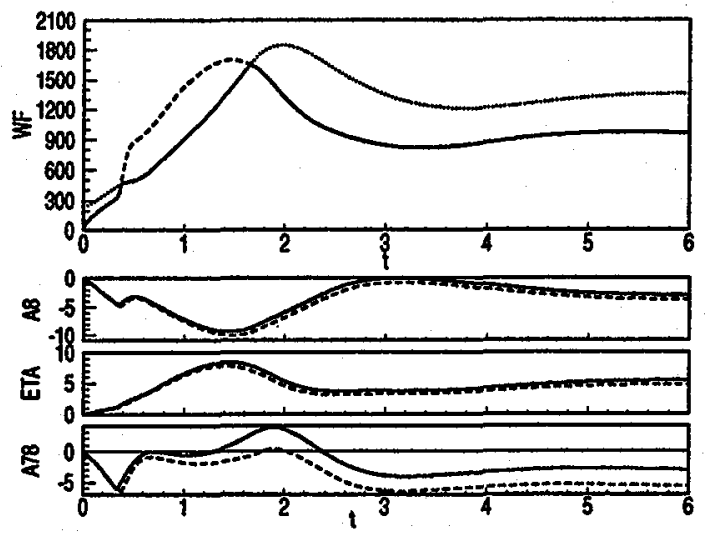

Figure 7: Comparison of controller commands with limited actuator commands for AW2 
desirable due to the wealth of existing linear analysis and design techniques. To this end, two linear representations of the limited system are now presented and discussed in regards to their use in antiwindup system analysis and design.

\section{Antiwindup Analysis}

The limited fuel flow and total area request can be linearly represented as resulting from diagonal (i.e. decoupled) input multiplicative perturbations to the corresponding plant actuators. By using the nonlinear simulation to determine bounds on the perturbations, the effects of the antiwindup design on stability and closed loop performance can be determined via $\mu$-analysis techniques.

\subsection{Limit Representation}

Fuel Flow Limit The multiplicative perturbation representation of the fuel flow limit is shown in Figure 8(a), where $W F \lim$ represents the limited value of the controller output, $W F_{-} c$. To obtain a bound on $\delta_{-} W F$, note from Figure $8(a)$ that $\delta_{-} W F=1-$ $W F_{-}$lim $/ W F_{-} c$. For the current step response simulations $\left(r_{\gamma}=3\right)$, this quantity reveals that $0 \leq$ $\delta_{-} W F<0.37$ for $\mathrm{AW} 1$ and $0 \leq \delta_{-} W F<0.44$ for AW2.

Total Area Limit Since the area limit is imposed on the total controller area request, this quantity is subject to multiplicative perturbation. As before, $\delta_{-} A=A \_\lim / A_{-} c-1$, with simulations showing $0 \leq \delta_{-} A<0.011$ for AW1 and $0 \leq \delta_{-} A<0.013$ for AW2. There remains the further issue of distributing the area perturbation among the three actuators. The amount of area correction is proportional to the relative sizes of each actuator; an accurate distribution of the total perturbed area can be based on the nominal actuator values. Thus $20 \%$ of the total area perturbation is distributed to each of the ETA and $A 8$ actuators, and $60 \%$ to the $A 78$ actuator, as illustrated in Figure $8(\mathrm{~b})$.

\section{$3.2 \mu$-Analysis}

Structured Singular Value The structured singular value provides an indication of the stability properties of a system subject to block diagonal perturbations. A standard form normalized perturbation $(\bar{\sigma}(\Delta)<1)$ representation of the limited STOVL closed loop system is shown in Figure 9, using the limit reformulation presented in the previous section to define a normalized perturbation and its inputs ( $q$ ) and outputs $(p)$. Robust stability to the multiplicative perturbations is guaranteed iff $\sup _{\omega} \mu\left(H_{q p}\right) \leq 1$
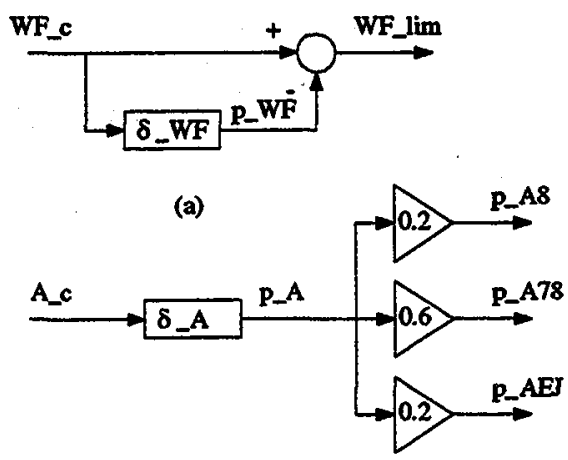

(b)

Figure 8: Multiplicative perturbation representation of limited controller fuel flow and area requests

[5]. Figure 10 depicts $\mu\left(H_{q p}\right)$ [2], obtained via bounding techniques, for the two antiwindup systems, AW1 and AW2. Note the larger stability margin of AW1 as compared with AW2. This illustrates the classical performance versus robustness tradeoff, in light of the inferior tracking performance of $\mathrm{AW} 1$ as seen in the simulations shown in Figure 4. A quantitative means of evaluating this performance degradation is now described.

Worst Case Bode Plots If robust stability is in fact achieved for the system under consideration, there remains the issue of performance degradation due to the perturbations. For small stability margins, performance can be degraded to such an extent that the system is unacceptable, even though it is robustly stable. Since the nominal $H_{\infty}$ control design is based on optimizing $\bar{\sigma}\left(H_{z w}\right)$, the quantity $\max _{\Delta}\left|H_{z_{i} w_{j}}^{p e r t}(j \omega)\right|$, the worst case bode plot, lends insight into the nature of the degradation in the frequency domain. This is possible using $\mu$-analysis by translating the nominal performance specifications into stability conditions using a so-called "performance block" [5].

The specific frequency response chosen to illustrate the performance difference between AW1 and AW2 is that from the $\gamma$ reference command to the weighted $\gamma$ tracking error, with the frequency weighting corresponding to the nominal design specifications (Figure 1). Conservative bounds [2] of the nominal and worst case responses are shown in Figure 11. Though both AW1 and AW2 are seen to have significant performance degradation with respect to the nominal system, AW1 exhibits particularly troublesome tracking behavior at low frequencies. This is consistent with the degraded behavior comparison made using the simulation responses of Figure 4.

It should be noted that in the above stability and performance analysis, conservatism is introduced by 


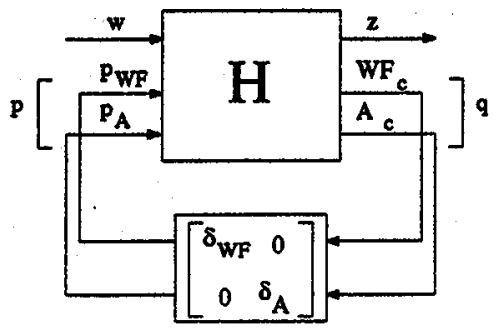

Figure 9: Standard perturbation form of limited STOVL closed loop system

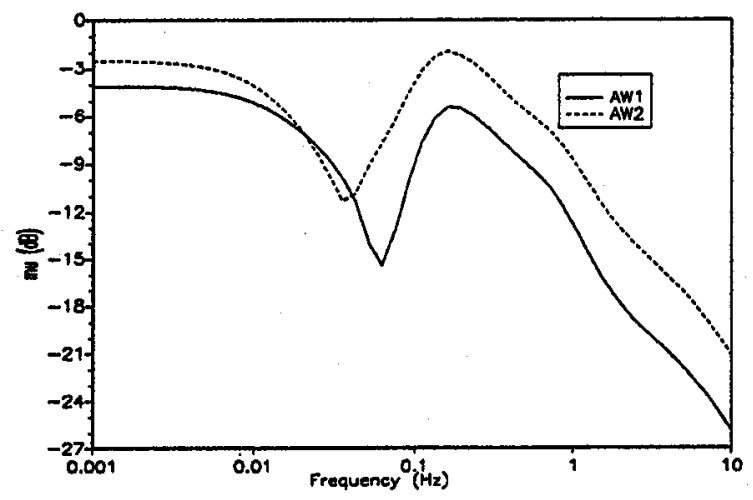

Figure 10: Structured singular value comparison for the limited STOVL closed loop systems

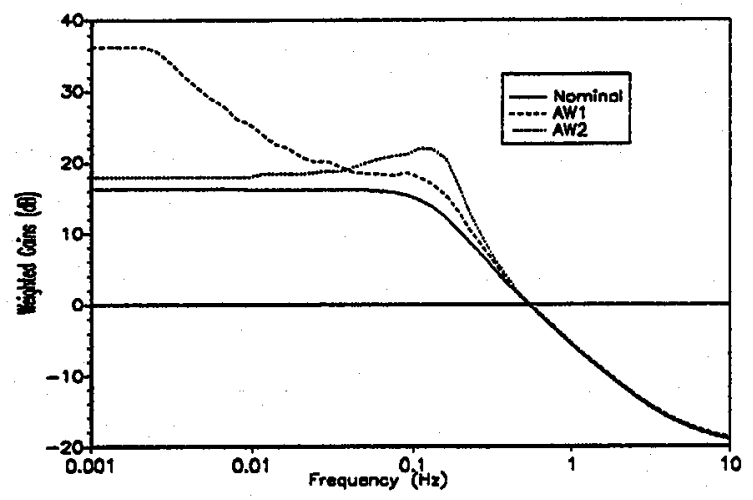

Figure 11: Nominal and worst case bode plots for the STOVL closed loop system

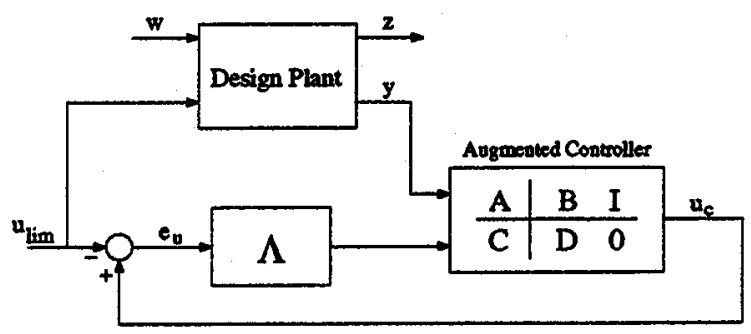

Figure 12: Antiwindup tracking system

using multiplicative perturbations to model the limited actuator commands. These models consider negative values of $\delta_{-} W F$ and $\delta_{-} A$ as "valid" perturbations, when in fact negative values for these perturbations were not generated by the simulation used to determine them. Thus if Figures 10 or 11 are generated assuming one or more negative perturbations, they are overly conservative.

The above analysis methods yield quantitative comparisons between competing antiwindup schemes. However, they do not lead to a design method since each $\Lambda$ requires a working nonlinear simulation to determine its corresponding perturbation bound. The following section addresses this issue.

\section{Proposed Design Approach}

The purpose of antiwindup protection is to provide an acceptable controller output during limited conditions. This goal is attained if the antiwindup protection prevents the "blowing up," or windup, of limited control signals. One way to accomplish this is to make the control signals track their limited values until they do not violate the limits. This philosophy effectively restates the antiwindup performance goal as a reference tracking problem. The limited signals serve as boundaries on the controller output which the antiwindup protection must enforce. This sections presents a framework by which this tracking characteristic of an antiwindup system can be quantified. The plausibility of this approach is illustrated by comparing the antiwindup systems AW1 and AW2.

In order to evaluate the limit tracking performance of an antiwindup system, the closed loop system of Figure 3 is modified by removing the limiting nonlinearity and treating the limited actuator signals as external reference inputs. The outputs of this new system are taken as the the corresponding controller signals. This view of the antiwindup system is shown in Figure 12. The antiwindup protection gain matrix, $\Lambda$, can now be viewed as a static controller, with the original augmented controller taking the role of the plant. The design plant appears in a feedforward configuration. 


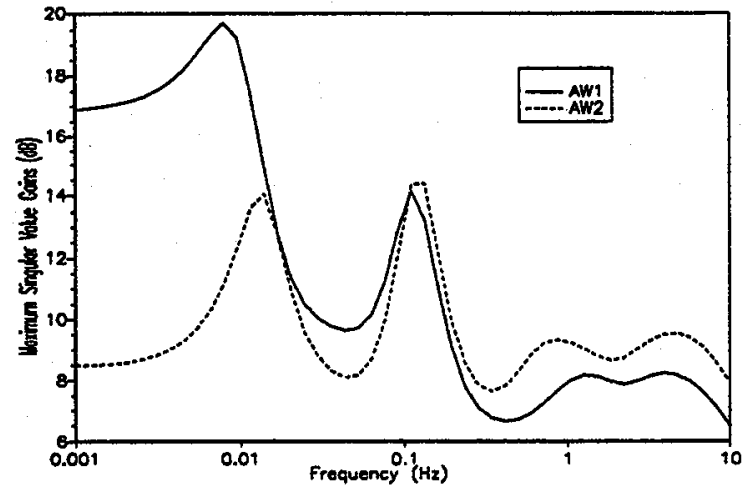

Figure 13: Comparison of antiwindup tracking performance criterion

In terms of this modified system, limit tracking performance will now be evaluated via the square transfer matrix from the reference inputs, $u_{l i m}$, to the tracking errors, $e_{u}$. Comparison between two competing antiwindup schemes is now possible without requiring a prior nonlinear simulation as in the previous section. This will be illustrated by examine this transfer function for the two antiwindup schemes AW1 and AW2.

Figure 13 compares the maximum singular value of the above square transfer matrix for AW1 (solid line) and AW2 (dashed line). It can be seen that AW1 suffers in limit tracking performance at low frequency compared with AW2. Furthermore, the frequency range of degraded tracking performance is very similar to that shown in the worst case bode plot of Figure 11. This suggests the possibility of a design method based on minimizing the infinity norm of the limit tracking transfer matrix.

\section{Conclusion}

The problems of actuator limits and antiwindup mechanisms are important considerations in any practical control design problem. This has been investigated here in the context of the integrated flight and propulsion control of a STOVL aircraft. A framework has been provided for the analysis of stability and performance degradation of a system operating under limited conditions. This is accomplished through the measures of structured singular value and worst case bode plots.

These measures provide the ability to quantitatively compare competing linear antiwindup schemes, as opposed to simply verifying their operation by viewing simulation time histories. This comparison is performed here on two antiwindup schemes having very different performance characteristics. It is shown that for these examples, the analysis results are consistent with the observed simulation behavior.
Finally, preliminary work is presented regarding the design of antiwindup systems. A cost criterion is proposed which is based on a fundamental transfer function of any LTI antiwindup system. It is shown to correctly identify deficiencies in the example systems, as identified in the above analysis methods. This suggests the plausibility of an optimization procedure based on this criterion. However, this procedure is one of determining an $H_{\infty}$ optimal static feedback gain-a problem for which there are currently few results.

\section{References}

[1] Garg, S., "Robust Integrated Flight/Propulsion Control Design for a STOVL Aircraft Using $H_{\infty}$ Control Design Techniques," Automatica, Vol.29, no.1, pp.129-145, 1993.

[2] "MATRIXx Robust Control Module, edition 2," Integrated Systems, Inc., Santa Clara, CA, June 1991.

[3] Campo, P.J., Morari, M., Nett, C.N., "Multivariable Anti-Windup and Bumpless Transfer: A General Theory," Proc. 1989 ACC, pp.1706-1711, 1989.

[4] Mattern, D., "A Linear, Multivariable Limit and Integrator Windup Protection Design Method," AIAA Guidance, Navigation, and Control Conference, 1993.

[5] Doyle, J. C., "Structured uncertainty in control system design," Proc. 1985 CDC, pp. 260-265. 
Public reporting burden for this collection of information is estimated to average 1 hour per response, including the time for reviewing instructions, searching existing data sources, gathering and maintaining the data needed, and completing and reviewing the collection of information. Send comments regarding this burden estimate or any other aspect of this collection of information, including suggestions for reducing this burden, to Washington Headquarters Services, Directorate for Information Operations and Reports, 1215 Jefferr (0) 20503.

\begin{tabular}{|l|c|c|}
\hline 1. AGENCY USE ONLY (Leave blank) & $\begin{array}{c}\text { 2. REPOAT DATE } \\
\text { April 1994 }\end{array}$ & $\begin{array}{r}\text { 3. REPOAT TYPE AND DATES COVERED } \\
\text { Final Contractor Report }\end{array}$ \\
\hline
\end{tabular}

\section{TITLE AND SUBTITLE}

Antiwindup Analysis and Design Approaches for MIMO Systems

\section{AUTHOR(S)}

Vincent R. Marcopoli and Stephen M. Phillips

WU-505-62-50

G-NAG3-1232

\section{PERFORMING ORGANIZATION NAME(S) AND ADDRESS(ES)}

8. PERFORMING ORGANIZATION REPORT NUMBER

Case Western Reserve University

Cleveland, Ohio 44106

9. SPONSORING/MONITORING AGENCY NAME(S) AND ADDRESS(ES)

10. SPONSORINGMONITORINC AGENCY REPORT NUMBER

National Aeronautics and Space Administration

Lewis Research Center

Cleveland, Ohio 44135-3191

NASA CR-195304

\section{SUPPLEMENTARY NOTES}

Project Manager, Walter Merrill, Instrumentation and Control Technology Division, organization code 2550, NASA Lewis Research Center, (216) 433-6328.

Unclassified - Unlimited

Subject Category 07

\section{ABSTRACT (Maximum 200 words)}

Performance degradation of multiple-input multiple-output (MIMO) control systems having limited actuators is often handled by augmenting the controller with an antiwindup mechanism, which attempts to maintain system performance when limits are encountered. The goals of this paper are: 1)To develop a method to analyze antiwindup systems to determine precisely what stability and performance degradation is incurred under limited conditions. It is shown that by reformulating limited actuator commands as resulting from multiplicative perturbations to the corresponding controller requests, $\mu$-analysis tools can be utilized to obtain quantitative measures of stability and performance degradation. 2)To propose a linear, time invariant (LTI) criterion on which to base the antiwindup design. These analysis and design methods are illustrated through the evaluation of two competing antiwindup schemes augmenting the controller of a Short Take-Off and Vertical Landing (STOVL) aircraft in transition flight.

\section{SUBJECT TERMS}

Feedback control; Controls; Actuator limits

15. NUMBER OF PAGES

16. PRICE CODE

$\mathrm{A} 02$

\begin{tabular}{|c|c|}
\hline 17. SECURITY CLASSIFICATION \\
OF REPORT \\
Unclassified
\end{tabular}$\quad \begin{gathered}\text { 18. SECURITY CLASSIFICATION } \\
\text { OF THIS PAGE } \\
\text { Unclassified }\end{gathered}$

19. SECURITY CLASSIFICATION OF ABSTRACT

Unclassified 